\title{
Progress in Brillouin slow light and its impact in fiber sensing
}

\author{
Miguel González Herráez, Kwang-Yong Song, Sang-Hoon Chin and Luc Thévenaz \\ Dept. of Electronics, Univ of Alcalá, Madrid, Spain. \\ and Nanophotonics and Metrology Lab. Ecole Polytechnique Federale de Lausanne, CH-1015, Switzerland \\ E-mail:miguelg@depeca.uah.es
}

\begin{abstract}
This paper reviews the major advances in Brillouin slow light devices along the last two years. We also focus on the positive and negative implications of this effect in the development of fiber sensors.

(C) 2006 Optical Society of America

OCIS codes: (999.9999) Slow light; (060.4370) Nonlinear optics, fibers; (290.5900) Scattering, Brillouin
\end{abstract}

\section{Introduction}

Slow light is known to be a very attractive approach to achieve all-optical delay lines and to provide a timing tool for photonic signal processing [1]. Successful experiments to control the light group velocity have been widely reported these past few years [2-8], showing the possibility to slow the speed of light up to nearly stopping it or to achieve group velocities exceeding the vacuum light velocity $c$. Most of these experiments use special media like cold atomic gases or electronic transitions in crystalline solids working at well defined wavelengths. A significant step towards real applications has been achieved last year when slow light was experimentally and efficiently demonstrated in optical fibres using stimulated Brillouin scattering [9] (see also [10,11]) and later other nonlinear interactions with larger natural bandwidth but reduced efficiency [12]. Among all these, the study of the Brillouin slow light effect has deserved much attention. The high flexibility of this interaction makes possible an active control of the speed of light in any type of fibre and at any wavelength, in particular in the low loss window of optical fibres. In this paper we review the recent progress in Brillouin slow light devices, with particular attention to the implications of the slow light effect in fiber sensing.

\section{Principle of Brillouin slow light}

The process of SBS is usually described as the interaction of two counterpropagating waves, a strong pump wave and a weak probe wave. If a particular phase matching condition is satisfied (namely $f_{\text {pump }}=f_{\text {probe }}+v_{B}, v_{B}$ being the Brillouin shift), an acoustic wave is generated which scatters photons from the pump to the probe wave, stimulating the process. SBS can be regarded as a narrowband amplification process, in which a strong pump wave produces a narrowband gain in a spectral region around $f_{\text {pump }}-v_{B}$ and a loss around $f_{\text {pump }}+v_{B}$. According to the Kramers-Kronig relation, a refractive index change is associated with the Brillouin gain/loss process and a substantial change of the group index $n_{g}=n+\omega d n / d \omega$ follows as a result of the sharp index transition (see Figure 1)

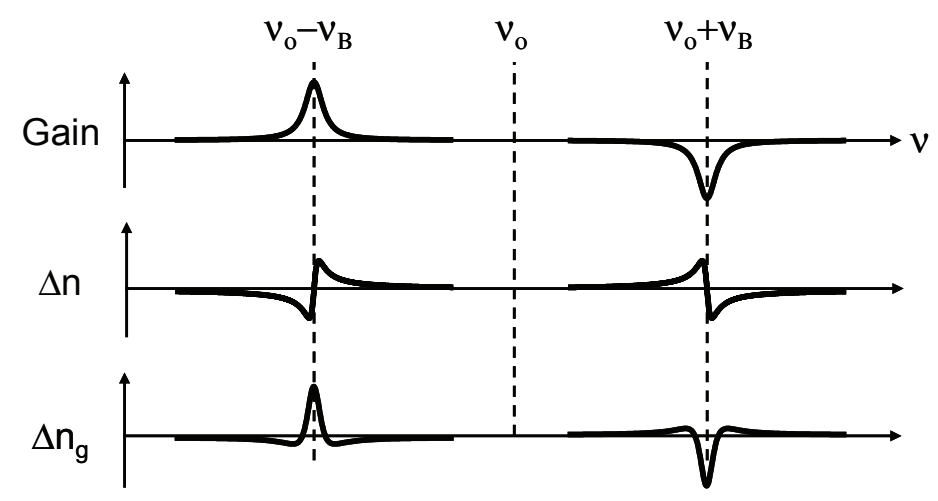

Fig. 1: Principle of Brillouin slow light. The narrowband gain/absorption processes caused by SBS in fibers induce sharp changes in the refractive index, which in turn cause strong variations of the group index. 


\section{TuC1.pdf}

Propagating at the peak of the gain resonance, a pulse experiences a logarithmic gain of $G=g_{B} I_{P} L_{e f f}$, where $g_{B}$ is the linear Brillouin gain coefficient, $I_{P}$ is the pump intensity and $L_{\text {eff }}$ is the effective length of the fiber. Simultaneously, the delay experienced by the signal is given by $\Delta \tau=G / 2 \pi \Delta v_{B}$, hence showing that the delay induced by the Brillouin interaction is larger for narrower resonances. A simple evaluation of these quantities in fibers shows that approximately $1 \mathrm{~ns}$ of delay is introduced per $\mathrm{dB}$ of gain suffered by the signal. The first experimental demonstrations of slow light using the SBS effect were independently achieved by two groups along last year [9][10]. In these experiments, the amount of delay achieved was limited to roughly $30 \mathrm{~ns}$ in several km-long fibers, and the group delay changes induced in the fiber were in the order of $10^{-3}$. In [15] it was experimentally demonstrated that arbitrarily large optically-controlled delays can be obtained by preventing pump depletion and amplified spontaneous Brillouin scattering. This simply required the insertion of unidirectional broadband attenuators in the signal path, leaving the pump path lossless. A more recent paper [16] has also shown that an extremely wide group velocity control in the fiber is possible using the same Brillouin principle, simply by using a shorter fiber and much higher pump powers. Group velocities as small as $71000 \mathrm{~km} / \mathrm{s}$, superluminal and even negative group velocities were observed. These experiments have been later extended to other kinds of fibers with highly improved efficiency [17][18].

\section{Spectral tailoring}

SBS has proved to be an unprecedented and unmatched flexible tool for the generation of slow light regarding its spectral tailoring capability. Indeed, a large variety of gain spectral profiles can be obtained by properly modulating the pump spectrum. When a perfectly coherent pump is used in the stimulated Brillouin interaction, the gain window appearing in the fiber transmission spectrum has a lorentzian shape whose characteristic spectral width is around 35 $\mathrm{MHz}$ in conventional single-mode fibers pumped at $1.55 \mu \mathrm{m}$. However, when the pump is modulated the gain bandwidth is given by the convolution of the pump spectrum and the Brillouin gain curve. Hence the effective Brillouin gain bandwidth $g(\Delta v)$ is given by:

$$
g(\Delta v)=P(\Delta v) \otimes g_{B}(\Delta v)
$$

where $\otimes$ denotes convolution, $P(\Delta v)$ is the normalized pump power spectral density and $g_{B}(\Delta v)$ is the characteristic lorentzian gain of the Brillouin amplification process (see Figure 2). As it can be seen, an adequate pump modulation can be used to change at will the gain spectrum of the Brillouin interaction. The simplest synthesized pump spectrum is a two-frequency spectrum with a frequency separation from 0 to $50 \mathrm{MHz}$, in the range of the natural Brillouin linewidth [19]. This results in overlapping gain profiles and eventually a reversed linear phase variation, so that fast light in gain regime was achieved. This modulation scheme makes also possible varying delays by changing the frequency separation rather than the pump power. It can be a more convenient solution in many practical implementations, the pump power remaining steady and stable.

A particularly useful case arises if the pump spectrum can also be approximated by a Lorentzian [20]. This pump spectrum can be created if the pump laser current is modulated with a noise signal, such as the output of a PRBS generator. In such conditions, the effective Brillouin gain shape is also a lorentzian whose width is the sum of the characteristic Brillouin gain width and the pump spectral width. In this particular case, the delay obtained as a function of the gain experienced by the signal is given by $\Delta \tau=G / 2 \pi\left(\Delta v_{B}+\Delta v_{P}\right)$, where $G$ is the logarithmic gain

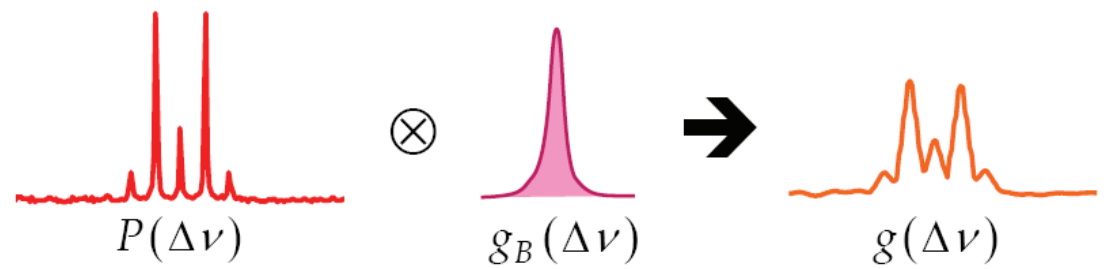

Fig. 2: Principle of spectral tailoring of Brillouin slow light devices. The effective gain spectrum created in the fiber results from the convolution of the pump spectrum and the characteristic gain spectrum of the Brillouin process. This is effectively translated into phase index changes that will affect notably the group velocity of the signal in the fiber. For broad pump spectra, the effective gain spectrum maps closely the pump spectrum. 


\section{TuC1.pdf}

suffered by the signal and $\Delta v_{P}$ is the pump spectral width. Thus, for the same amount of signal gain, a tenfold increase of the bandwidth of the interaction comes at the expense of a tenfold reduction of the achieved delay. Since $G=g_{B} I_{P} L_{\text {eff }} \Delta v_{B} /\left(\Delta v_{B}+\Delta v_{P}\right)$, achieving the same absolute delay with a tenfold increase in the bandwidth of the interaction requires a 100 -fold increase in the power of the pump or the effective length. More importantly, however, in terms of fractional delay (i.e. the delay divided by the pulse length) the same fractional delay with a tenfold increase in the bandwidth can only be achieved with a tenfold increase of the pump power. This technique was ultimately extended to achieve 12-GHz bandwidth slow light [21].

\section{Potential benefits and drawbacks in fiber sensing}

Slow light devices offer new and unique possibilities in fiber sensing. As it has been pointed out before, optical fiber slow light should enable the development of all-optical delay lines with no mechanical movement, featuring higher stability and compactness than other electro-mechanical solutions. It must be pointed out that a slow light effect can be activated only in a spectral resonance, thus on a limited optical frequency span given by the resonance width. This makes definitely slow light delay lines dedicated to signals with a limited bandwidth and totally impracticable for length scanning in low coherence interferometry, for instance. Group velocity engineering of different signals in the same optical medium can also be of interest, for instance to achieve phase matching between signals at different wavelengths propagating in the same fiber. Slow light can also be used to increase the effective interaction length of light and matter, for example in evanescent-wave sensors. Beyond this, some new and exciting applications of slow light are emerging, that may be profitable for fiber sensing in the future. For instance, it has been demonstrated that ultraslow group velocities can enhance significantly nonlinear interactions, enabling nonlinear processes with extremely weak optical fields [22][23]. The most striking possibilities arise when the group velocity of light is rendered low enough to be in the order of the sound velocity in the material. In these conditions, strong coupling between light and sound can be achieved, and new phenomena like anomalous stimulated Brillouin scattering (forward SBS) can be obtained with high efficiency [24].

A fundamental drawback of the slow light effect is a time biasing that can be observed in distributed sensors based on the Brillouin interaction. Distributed fiber-optic sensors offer unique capabilities for the monitoring of magnitudes over long distances. In many applications, these sensors avoid the need of thousands of pin-point sensors and complicated multiplexing schemes. Among these, distributed Brillouin sensors have attracted much research interest in the past years [25-30], and are now widely used for the monitoring of strain and temperature distribution within large structures in civil engineering [26]. Several techniques have been proposed for performing distributed Brillouin sensing, and the principal configurations have been summarized in Figure 3. In the Brillouin Optical Time Domain Reflectometer (BOTDR) configuration [27], a pump pulse is launched into the fibre and the spontaneous Brillouin backscattered light is synchronously analysed as a function of the time (distance along the fiber) using heterodyne detection. For each position, the pump-Stokes frequency shift is determined, which is then translated into strain or temperature values. In the Brillouin Optical Time Domain Analysis configuration, however, the Brillouin interaction is performed in the stimulated regime, thus requiring the need of two counterpropagating waves, a powerful pulsed pump and a weak cw probe (BOTDA-1) [28] or a powerful continuous-wave pump and a weak pulsed probe (BOTDA-2) [29]. The frequencies of pump and probe waves are set so as to have Brillouin gain/attenuation in the probe, and the changes in the Brillouin shift along the fiber (typically related to strain or temperature variations) yield a time-dependent variation of the detected probe signal in the pump end. Similar

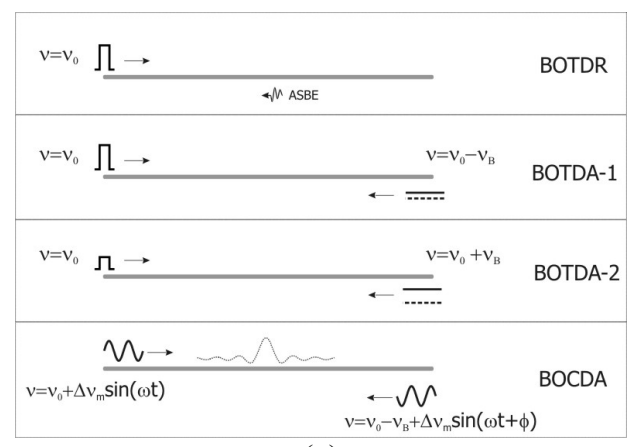

(a)

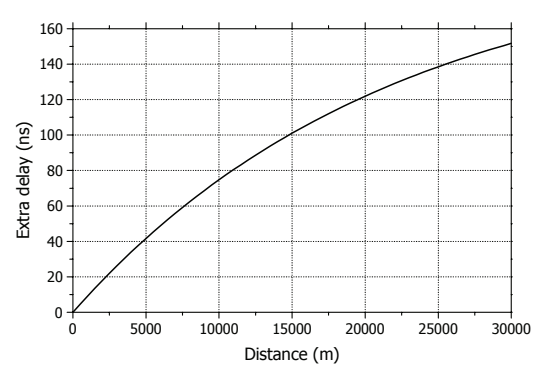

(b)

Fig. 3: Time biasing of distributed Brillouin sensors. (a) Typical configurations for distributed Brillouin sensing. (b) Probe pulse delaying as a function of distance for the BOTDA-2 configuration, pump power is $6 \mathrm{~mW}$. 


\section{TuC1.pdf}

schemes to BOTDA-1 and BOTDA-2 can be developed in the frequency domain. Lastly, centimetric resolution sensors make use of the Brillouin Optical Correlation Domain Analysis (BOCDA) technique [30], in which the short correlation length between two modulated, counterpropagating laser beams is exploited to achieve very small amplification windows whose width and position is controlled by the modulation parameters.

All the sensing schemes developed up to now have estimated their resolution by using directly the pulse width (for BOTDA and BOTDR) or the calculated correlation window for BOCDA. However, in these approaches, the group velocity changes induced by the Brillouin amplification/attenuation mechanisms have been fully neglected. We have recently pointed out that the slow light effect can have a particularly high impact in the BOTDA-2 configuration [31]. In these systems, the slow light effect does not only introduce a time biasing of the trace, but also a strong impairment in the available resolution due to the fact that this time-biasing is strongly dependent on the pump-probe frequency difference. A frequency-dependent correction of the time axis should be sufficient to overcome this resolution impairment, but this correction does not seem straightforward, and cumulative errors might result from a multi-pass correction algorithm.

\section{Conclusion}

We have reviewed the progress in Brillouin slow light devices along the last two years. We have explained the basic principle underlying slow light using SBS and we have reviewed the main advances in the spectral tailoring of these devices. We have pointed out some implications of this effect that may be envisaged in fiber sensors and this field is still widely unexplored. Certainly very innovative solutions will emerge in the coming years using the unique features of this novel photonic tool.

We acknowledge support from the Swiss National Science Foundation through project 200021-109773/1. MGH acknowledges support from the University of Alcalá through project PI2005/076 and Comunidad de Madrid through project FUTURSEN S-0505/AMB/0374.

\section{References}

R. W. Boyd and D. J. Gauthier, in Progress in Optics, edited by E. Wolf, Vol. 43, Chap. 6, p.497.

[2] A. M. Akulshin, S. Barreiro, and A. Lezema, Phys. Rev. Lett. 83, 4277 (1999).

[3] M. D. Stenner, D. J. Gauthier, and M. A. Neifeld, Nature (London) 425, 695 (2003).

[4] L. V. Hau, S. E. Harris, Z. Dutton, and C. H. Behroozi, Nature (London) 397, 594 (1999).

[5] C. Liu, Z. Dutton, C. H. Behroozi, and L. V. Hau, Nature (London) 409, 490 (2001).

[6] L. J. Wang, A. Kuzmich, and A. Dogariu, Nature (London) 406, 277 (2000).

[7] M. S. Bigelow, N. N. Lepeshkin, and R. W. Boyd, Science 301, 200 (2003).

[8] P. C. Ku, F. Sedgwick, C. J. Chang-Hasnain P. Palinginis, T. Li, H. L. Wang, S. W. Chang and S. L. Chuang, Opt. Lett. 292291 (2005).

[9] K. Y. Song, M. G. Herráez, and L. Thévenaz, Opt. Express 13, 82-88 (2005).

[10] Y. Okawachi, M.S. Bigelow, J. E. Sharping, Z. Zhu, A. Schweinsberg, D.J. Gauthier, R.W. Boyd, and A.L. Gaeta, Phys. Rev. Lett. 94 $153902(2005)$.

[11] J. T. Mok and B. J. Eggleton "Expect more delays" Nature 433811 (2005)

[12] J. E. Sharping, Y. Okawachi, and A. L. Gaeta, Opt. Express 13, 6092 (2005).

[13] D. Dahan and G. Eisenstein, Opt. Express 13, 6234 (2005)

[14] J. E. Sharping, Y. Okawachi, J. van Howe, C. Xu, Y. Wang, A. E. Willner, and A. L. Gaeta, Opt. Express 13, 7872 (2005).

[15] K. Y. Song, M. G. Herráez, and L. Thévenaz, Opt. Lett. 30, 1782 (2005)

[16] M. González-Herráez, K.Y. Song and L. Thévenaz, Appl. Phys. Lett. 87, 081113 (2005)

[17] C. Jauregui, H. Ono, P. Petropoulos and D. J. Richardson, postdeadline paper PDP2, OFC 2006, Anaheim, CA, Mar. 5-10, 2006.

[18] K. S. Abedin, K. Y. Song, L. Thevenaz, M. G. Herraez and K. Hotate, postdeadline paper CPDA9, CLEO 2006, Long Beach CA, May 2126, 2006.

[19] K. Y. Song, M. G. Herráez, and L. Thévenaz, Opt. Express. 13, 9758 (2005)

[20] M. González-Herráez, K.Y. Song and L. Thévenaz, Opt. Express. 14, 1395 (2006).

[21] Z. Zhu, A.M.C. Dawes, D.J. Gauthier, L. Zhang, and A.E. Willner, postdeadline paper PDP1, OFC 2006, Anaheim, CA, Mar. 5-10, 2006.

[22] M. M. Kash, V. A. Sautenkov, A. S. Zibrov, L. Hollberg, G. R. Welch, M. D. Lukin, Y. Rostovtsev, E. S. Fry and M. O. Scully, Phys. Rev. Lett. 825229 (1999)

[23] M. Soljacic and J. D. Joannopoulos, Nature Materials 3, 211 (2004)

[24] A. B. Matsko, Y. V. Rostovtsev, H. Z. Cummins and M. O. Scully, Phys. Rev. Lett. 845752 (2000).

[25] T. Horiguchi and M. Tateda, Opt. Lett. 14, 408 (1989)

[26] L. Thévenaz, M. Facchini, A. Fellay and P. Robert, in 13th International Conference on Optical Fiber Sensors, B. Y. Kim and K.Hotate, eds., Proc. SPIE 3746, 343 (1999)

[27] S. M. Maughan, H. H. Kee and T. P. Newson, Opt. Lett. 26, 331 (2001)

[28] M. Niklès, L. Thévenaz, P. A. Robert, Opt. Lett. 21, 758 (1996)

[29] X. Bao, D. J. Webb and D. Jackson, Opt. Lett. 18, 1561 (1993)

[30] K. Hotate and M. Tanaka, IEEE Photon. Technol. Lett. 14, 179 (2002).

[31] L. Thevenaz, K. Y. Song and M. G. Herraez Opt. Lett. 31715 (2006) 